\title{
DIVISION XII / COMMISSION 14 / WORKING GROUP SOLIDS AND THEIR SURFACES
}

\author{
CHAIR \\ Gianfranco Vidali
}

SEXENNIAL REPORT 2003 - 2009

\section{Introduction}

In the last decade there has been a tremendous increase of interest in studying processes occurring on IS dust. In part this is due to the availability of ground-based and spaceborne high quality instruments which have been used to detect molecules in diverse astrophysical environments, from protoplanetary disks to hot cores and dense clouds. It has also been recognized that IS dust has an important role in the formation of molecules, from molecular hydrogen to methanol. Therefore, it is necessary not to study only properties of dust, but also understand how atoms and molecules interact with and on dust.

This has prompted a number of laboratories with a tradition of working in surface science to study the processes associated with dust. Besides the standard probes that have been used in the past, now there are available techniques that can give precise information at the atomic/molecular level about the formation of molecules on dust. For instance, Thermal Programmed Desorption (TPD), Reflection Absorption Infrared Spectrometry (RAIRS), Resonant Enhanced Multiphoton Ionization (REMPI), and Atom Force Microscopy (AFM) give information about the kinetics and energetics of diffusion of atoms/molecules on and desorption from surfaces, the products of reaction, the ro-vibrational state of ejected products, and the morphology of the solid surfaces, respectively. One of the consequences of the interest by surface science laboratories in studying physical/chemical properties of dust analogues and reactions occurring on them is that works of interest to astrochemistry are now regularly published in chemical physics/ surface science journals such as J. Chem. Phys., J. Phys. Chem., Phys. Chem. Chem. Phys., Surface Science, and others.

While in the past there has been a large number of laboratory studies of the interaction of charged particles and radiation with ice-covered dust grain analogues, most recent work points at new directions of research that will likely continue to be studied in the near future, i.e. the formation of molecules in/on ices by hydrogenation reactions, the properties of mixed ices, and the formation and properties of dust particles, including nanoparticles. Observations with ALMA, SOFIA and Herschel will yield more detailed information on dust and molecules, and theoretical studies will need to sort out the role of dust particles in molecule formation.

\section{Meetings}

Sessions about atomic/molecular interaction with surfaces are often featured at regularly scheduled COSPAR, AAS and Lunar and Planetary Institute meetings. For more 
information about these meetings, visit the Web sites of the respective organizations. For information about the meetings below, visit the web site of the Canadian Astronomy Data Centre (Web link: <www1.cadc-ccda.hia-iha.nrc-cnrc.gc.ca/meetings/>). Unfortunately, a number of meetings' official web sites have been taken down.

Most important meetings (listed in inverse chronological order):

- Cosmic Dust - Near And Far, Heidelberg, Germany, 8-12 September 2008

- Bridging the Laboratory and Astrophysics, 212th AAS, St. Louis, MO, USA, 1-5 June 2008

- The Molecular Universe: International Meeting on the Physics and Chemistry of the IS Medium, Arcachon, France, 5-8 May 2008

- AbSciCon 2008: Fifth Astrobiology Science Conference, Santa Clara, CA, USA, 1517 April 2008

- Titan Observations, Experiments, Computations, and Modeling, Miami, FL, USA, 24-26 March 2008

- Organic Matter in Space, IAU Symposium No. 251, Hong Kong, 18-22 February 2008

- The Evolving Insterstellar Medium in the Milky Way and Nearby Galaxies, Pasadena, CA, USA, 2-5 December 2007

- Bioastronomy 200\%: Molecules, Microbes, and Extraterrestrial Life, San Juan, PR, USA, 16-20 July 2007

- Origins of Solar Systems, 2007 Gordon Conference, South Hadley, MA, USA, 8-13 July 2007

- Molecules in Space and Laboratory, Paris, France, 14-18 May 2007

- Astronomy in the Submillimeter and Far Infrared Domains with the Herschel Space Observatory, Les Houches Winter School, France, 23 April - 4 May 2007

- Titan Observations, Experiments, Computations, and Modeling, Honolulu, HI, USA, 5-7 February 2007

- Science with ALMA: a New Era for Astrophysics, Madrid, Spain, 13-16 November 2006

- IS Medium, Heidelberg Summer School, Germany, 25-29 September 2006

- From Dust to Planetesimals, Ringberg Castle, Bavaria, Germany, 11-15 September 2006

- Cosmic Chemistry and Molecular Astrophysics, Nobel Symposium, Sudertuna, Sweden, 10-15 June 2006

- Complex Molecules in Space - present Status and Prospects with ALMA, Fuglsocentret, Denmark, 8-11 June 2006

- Carbon in Space, International workshop, Lago di Como, Italy, 22-25 June 2006

- NASA Laboratory Astrophysics Workshop, Las Vegas, NV, USA, 14-16 February 2006

- Astrochemistry - A Molecular Approach, Honolulu, HI, USA, 17-18 December 2005

- Hunt for Molecules, Paris, France, 19-20 September 2005

- Protostars and Planets. V, Big Island, HI, USA, 24-28 October 2005

- 5th. European Workshop on Astrobiology, Budapest, Hungary, 10-12 October 2005

- Astrochemistry throughout the Universe: Recent Successes and Current Challenges, IAU Symposium No. 231, Monterey, CA, USA, 29 August - 2 September 2005

- Astrobiology and the Origins of Life, Hamilton, Canada, 24- May -10 June 2005

- The Spitzer Space Telescope: New Views of the Cosmos, Pasadena, CA, USA, 9-12 November 2004

- The Dusty and Molecular Universe: A prelude to Herschel and ALMA, Paris, France, 27-29 October 2004 
- Effects of Space Radiation on Solar System Ices, AOGS 2004 Session SP2, Singapore, 5-9 July 2004

- Astrophysics of Dust, Estes Park, CO, USA, 2003

Published works in the area of molecular reactions on solid surfaces have been sorted in 4 sections:

(a) reviews

(b) observations of dust and ices in the ISM

(c) dust (formation, properties, and exposure to space environment)

(d) interactions of atoms and molecules with solids in simulated ISM conditions

(e) interaction of radiation and charged particles with ices in simulated ISM conditions

Obviously, there is a certain degree of arbitrariness in the sorting. Several papers could be entered in more than one section. The papers listed here are the ones that appeared in print since the last review by the Working Group on Molecular Reactions on Solid Surfaces in 2002; therefore, this report covers a six-year period. Works are listed in inverse chronological order.

\section{Reviews}

\section{References}

Charnley, S. B. \& Rodgers, S. D. 2008, SSRv, 40. IS reservoirs of cometary matter

Williams, D. A., Brown, W. A., Price, S. D., et al. 2007, Astron. E Geophys., 48, 25. Molecules, ices and astronomy

Tothill, N. F. H. 2007, EAS-PS, 25, 327. AST/RO: lessons from a decade of sub-mm astronomy at the South Pole

Slavin, J. D. \& Frisch, P. C. 2007, SSRv, 130, 409. The chemical composition of IS matter at the solar location

Herbst, E. \& Cuppen, H. M. 2006, PNAS, 103, 12257. IS chemistry special feature: Monte Carlo studies of surface chemistry and nonthermal desorption involving IS grains

Dartois, E. 2005, SSRv, 119, 293. The ice survey opportunity of ISO.

Abergel, A., Verstraete, L., Joblin, C., et al. 2005, SSRv, 119, 247. The cool IS medium

Molster, F. \& Kemper, C. 2005, SSRv, 119, 3. Crystalline silicates

Grün, E., Srama, R., Krüger, H., et al. 2005, Icarus, 174, 1. Dust astronomy. 2002 Kuiper Prize lecture

van Dishoeck, E. F. 2004, ARAA 42, 119. ISO spectroscopy of gas and dust - from molecular clouds to protoplanetary disks

Clayton, D. D., Nittler, L. R. 2004, ARAA, 42, 39. Astrophysics with presolar stardust

Dorschner, J. 2003, Astromineralogy 609, 1. From dust astrophysics towards dust mineralogy - a historical review

Draine, B. T. 2003, ARAA 41, 241. IS dust grains

Williams, D. A. \& Viti, S. 2002, Ann. Rep. Prog. Chem. Sect. C 98, 87. Recent progress in astrochemistry

\section{Observations of dust and ices in the ISM}

\section{References}

Öberg, K. I., Boogert, A. C. A., Pontoppidan, K. M., et al. 2008, ApJ, 678, 1032. The c2d Spitzer spectroscopic survey of ices around low-mass young stellar objects. III. $\mathrm{CH}_{4}$

Pontoppidan, K. M., Boogert, A. C. A., Fraser, H. J., et al. 2008, ApJ, 678, 1005. The c2d Spitzer spectroscopic survey of ices around low-mass young stellar objects. II. $\mathrm{CO}_{2}$

Boogert, A. C. A., Pontoppidan, K. M., Knez, C., et al., 2008, ApJ, 678, 985. The c2d Spitzer spectroscopic survey of ices around low-mass young stellar objects. I. $\mathrm{H}_{2} \mathrm{O}$ and the 5-8 $\mu \mathrm{m}$ bands 
Sonnentrucker, P., Neufeld, D. A., Gerakines, P. A., et al. 2008, ApJ, 672, 361-370. Fully sampled maps of ices and silicates in front of Cepheus A East with the Spitzer Space Telescope

Li, Y., Li, A., \& Wei, D. M. 2008, ApJ, 678, 1136. Determining the dust extinction of gamma-ray burst host galaxies: a direct method based on optical and $X$-ray photometry

Hough, J. H., Aitken, D. K., Whittet, D. C. B., et al. 2008, MNRAS, 387, 797. Grain alignment in dense IS environments: spectropolarimetry of the 4.67- $\mu \mathrm{m} C O$-ice feature in the field star Elias 16 (Taurus dark cloud)

Sirocky, M. M., Levenson, N. A., Elitzur, M., et al. 2008, ApJ, 678, 729. Silicates in ultraluminous infrared galaxies

Berné, O., Joblin, C., Rapacioli, M., et al. 2008, A $\mathscr{E A}$ (Letters), 479, L41. Extended red emission and the evolution of carbonaceous nanograins in NGC 7023

Li, Y., Hopkins, P. F., Hernquist, L., et al. 2008, ApJ, 678, 41. Modeling the dust properties of $z \simeq 6$ quasars with ART $T^{2}$ - All-wavelength Radiative Transfer with Adaptive Refinement Tree

Nozawa, T., Kozasa, T., Habe, A., et al. 2008, in: Origin of Matter and Evolution of Galaxies, AIP-CP, 1016, 55. Evolution of dust in primordial supernova remnants and its influence on the elemental composition of hyper-metal-poor stars

Dwek, E., Arendt, R. G.; Bouchet, P., et al. 2008, ApJ, 676, 1029. Infrared and X-Ray evidence for circumstellar grain destruction by the blast wave of Supernova $1987 \mathrm{~A}$

Draine, B. T., Dale, D. A., Bendo, G., et al. 2007, ApJ, 663, 866. Dust masses, PAH sbundances, and starlight intensities in the SINGS galaxy sample

Angeloni, R., Contini, M., Ciroi, S., \& Rafanelli, P. 2007, AJ, 134, 205. Silicates in D-Type symbiotic stars: an Infrared Space Observatory overview

Mason, R. E., Wright, G. S., Adamson, A., \& Pendleton, Y. 2007, ApJ, 656, 798. Spectropolarimetry of the $3.4 \mu \mathrm{m}$ absorption feature in NGC 1068

Terada, H., Tokunaga, A. T., Kobayashi, et al. 2007, ApJ, 667, 303. Detection of water ice in edge-on protoplanetary disks: HK Tauri $B$ and $H V$ Tauri $C$

Quanz, S. P., Henning, T., Bouwman, J., et al. 2007, ApJ, 668, 359. Evolution of dust and ice features around FU Orionis objects

Whittet, D. C. B., Shenoy, S. S., Bergin, E. A., et al. 2007. ApJ, 655, 332. The abundance of carbon dioxide ice in the quiescent intracloud medium

Markwick-Kemper, F., Gallagher, S. C., Hines, D. C., \& Bouwman, J. 2007, ApJ (Letters), 668, L107. Dust in the wind: crystalline silicates, corundum, and periclase in $P G 2112+059$

Bottinelli, S., Boogert, A. C. A., van Dishoeck, E. F., Oberg, K., et al. 2007, in: J. L. Lemaire \& F. Combes (eds.), Molecules in Space and Laboratory (Paris: S. Diana), p. 11. $\mathrm{NH}_{3}$ and $\mathrm{CH}_{3} \mathrm{OH}$ in ices surrounding low-mass $\mathrm{YSO}$

Bai, L., Rieke, G. H., \& Rieke, M. J. 2007, ApJ (Letters), 668, L5. A search for infrared emission from intracluster dust in Abell 2029

Davis, S. S. 2007, ApJ, 660, 1580. Ice formation in radiated accretion disks

Ellison, S. L., Prochaska, J. X., \& Lopez, S. 2007, MNRAS, 380, 1245. The Galactic deuterium abundance and dust depletion: insights from an expanded Ti/H sample

Vollmer, C., Hoppe, P., Brenker, F. E., \& Holzapfel, C. 2007, ApJ (Letters), 666, L49. Stellar $\mathrm{MgSiO}_{3}$ perovskite: a shock-transformed stardust silicate found in a meteorite

Bacmann, A., Lefloch, B., Parise, B., et al. 2007, in: J. L. Lemaire \& F. Combes (eds.), Molecules in Space and Laboratory (Paris: S. Diana), p. 9. Methanol and deuterium fractionation in pre-stellar cores

Maiolino, R. 2007, in: K. A. van der Hucht (ed.), Highlights of Astronomy, 14, 262. Dust at $z>6$. Observations and theory

Andersson, B.-G. \& Potter, S. B. 2007, ApJ 665, 369. Observational constraints on IS grain alignment

Sujatha, N. V., Murthy, J., Shalima, P., \& Henry, R. C. 2007, ApJ, 665, 363. Measurement of dust optical properties in the Coalsack nebula

Kulkarni, V. P., York, D. G., Vladilo, G., \& Welty, D. E. 2007, ApJ (Letters), 663, L81. 9.7 $\mu m$ silicate absorption in a damped Ly a absorber at $z=0.52$

Bethell, T. J., Chepurnov, A., Lazarian, A., \& Kim, J. 2007, ApJ, 663, 1055. polarization of dust emission in clumpy molecular clouds and cores 
Dwek, E., Galliano, F., \& Jones, A. P. 2007, ApJ, 662, 927. The evolution of dust in the early Universe with applications to the galaxy SDSS J1148+5251

Dessauges-Zavadsky, M., Combes, F., \& Pfenniger, D. 2007, A\&SA 473, 863. Molecular gas in high-velocity clouds: revisited scenario

Bisschop, S. E., Jørgensen, J. K., van Dishoeck, E. F., \& de Wachter, E. B. M. 2007, A $\mathscr{E} A$, 465, 913. Testing grain-surface chemistry in massive hot-core regions

Inoue, A. K., Buat, V., Burgarella, D., et al. 2006, MNRAS, 370, 380. Effects of dust scattering albedo and 2175- $\AA$ bump on ultraviolet colours of normal disc galaxies

Colangeli, L. 2006, MemSAIS, 9, 161. Measurement of dust properties in different solar system environments

Nozawa, T., Kozasa, T., \& Habe, A. 2006, ApJ, 648, 435. Dust destruction in the high-velocity shocks driven by supernovae in the early Universe

Spoon, H. W. W., Tielens, A. G. G. M., Armus, L., et al. 2006, ApJ, 638, 759. The detection of crystalline silicates in ultraluminous infrared galaxies

Sofia, U. J., Gordon, K. D., Clayton, G. C., et al. 2006, ApJ, 636, 753. Probing the dust responsible for Small Magellanic Cloud extinction

Huard, T. L., Pontoppidan, K. M., Boogert, A., et al. 2006, BAAS, 38, 1055. Variations in the extinction law, ice abundance, and dust grains in molecular cloud cores

Chiar, J. E., Pendleton, Y., Ennico, K., et al. 2006, BAAS, 38, 1013. The non-linear relationship between silicate absorption depth and IR extinction in dense clouds

Huebner, W. F. \& Snyder, L. E. 2006, in: Comets and the Origin and Evolution of Life (Springer), p.113. Macromolecules: from star-forming regions to comets to the origins of life

Prieto-Ballesteros, O., Kargel, J. S., Fernández-Sampedro, M., et al. 2005, Icarus, 177, 491. Evaluation of the possible presence of clathrate hydrates in Europa's icy shell or seafloor

Knez, C., Boogert, A. C. A., Pontoppidan, K. M., Kessler-Silacci, J., et al. 2005. ApJ (Letters), 635, L145. Spitzer mid-infrared spectroscopy of ices toward extincted background stars.

Velusamy, T., Langer, W. D., \& Willacy, K. 2005, in: D. C. Lis, G. A. Blake \& E. Herbst (eds.), Astrochemistry throughout the Universe: Recent Successes and Current Challenges, Proc. IAU Symposium No. 231 (Cambridge: CUP), p. 137. $\mathrm{CO}_{2}$ ice in cold dense dark cloud cores: abundance vs. visual extinction

Lee, J. C. \& Ravel, B. 2005, in: X-ray Diagnostics of Astrophysical Plasmas: Theory, Experiment, and Observation AIP-CP, 774, 255. Prospects for determining the grain composition of the IS medium with Chandra and Astro E2.

Bernstein, M. P., Sandford, S. A., \& Allamandola, L. J. 2005, ApJS, 161, 53. The mid-infrared absorption spectra of neutral polycyclic aromatic hydrocarbons in conditions relevant to dense IS clouds

Ruiterkamp, R., Cox, N. L. J., Spaans, M., et al. 2005, A\&A, 432, 515. PAH charge state distribution and DIB carriers: implications from the line of sight toward HD 147889

Massey, P., Plez, B., Levesque, E. M., et al. 2005, ApJ, 634, 1286. The reddening of red supergiants: when smoke gets in your eyes

Andrews, S. M. \& Williams, J. P. 2005, ApJ, 631, 1134. Circumstellar dust disks in TaurusAuriga: the sub-millimeter perspective

Stratta, G., Perna, R., Lazzati, D., et al. 2005, NCimC 28, 693. Dust extinction properties of a sample of bright $X$-rays afterglows

del Burgo, C. \& Laureijs, R. J. 2005, MNRAS, 360, 901. New Insights into the dust properties of the Taurus molecular cloud TMC-2 and its surroundings

Joblin, C., Abergel, A., Bernard, J.-P., et al. 2005, in: D. C. Lis, G. A. Blake \& E. Herbst (eds.), Astrochemistry throughout the Universe: Recent Successes and Current Challenges, Proc. IAU Symposium No. 231 (Cambridge: CUP), p. 194. Very small particles and chemistry in photo-dissociation regions: from ISO to Spitzer

Remijan, A. J., Hollis, J. M., Lovas, F. J., et al. 2005, ApJ, 632, 333. IS isomers: the importance of bonding energy differences

Gerakines, P. A., Bray, J. J., Davis, A., \& Richey, C. R. 2005, ApJ, 620, 1140. The strengths of near-infrared absorption features relevant to IS and planetary ices

Snell, R. L., Hollenbach, D., Howe, J. E., et al. 2005, ApJ, 620, 758. Detection of water in the shocked gas associated with IC 443: constraints on shock models 
Pontoppidan, K. M. \& the c2d Team 2005, in: D. C. Lis, G. A. Blake \& E. Herbst (eds.), Astrochemistry throughout the Universe: Recent Successes and Current Challenges, Proc. IAU Symposium No. 231 (Cambridge: CUP), p. 319. The spatial distribution of ices in star-forming regions

van Boekel, R., Min, M., Waters, L. B. F. M., et al. 2005, A\&SA, 437, 189. A $10 \mu m$ spectroscopic survey of herbig Ae star disks: grain growth and crystallization

Duchêne, G., McCabe, C., Ghez, A. M., \& Macintosh, B. A. 2004, ApJ, 606, 969. A multiwavelength scattered light analysis of the dust grain population in the GG Tauri circum-binary ring

Jones, A. P., d'Hendecourt, L. B., Sheu, S.-Y., et al. 2004, A\&A, 416, 235. Surface C-H stretching features on meteoritic nanodiamonds

Pendleton, Y. J. 2004, in: A. N. Witt, G. C. Clayton \& B. T. Draine (eds.), Astrophysics of Dust ASP-CS, 309, 573. Hydrocarbons in meteorites, the Milky Way, and other galaxies

Bot, C., Boulanger, F., Lagache, et al. 2004, A\&GA, 423, 567. Multi-wavelength analysis of the dust emission in the Small Magellanic Cloud

Ehrenfreund, P., Fraser, H. J., Blum, J., et al. 2003, PESSS, 51, 473. Physics and chemistry of icy particles in the Universe: answers from microgravity

Johnstone, D., Fiege, J. D., Redman, R. O., et al. 2003, ApJ (Letters), 588, L37. The G11.110.12 infrared-dark cloud: anomalous dust and a non-magnetic isothermal model

Wolf, S., Padgett, D. L., \& Stapelfeldt, K. R. 2003, ApJ, 588, 373. The circumstellar disk of the butterfly star in Taurus.

Kimura, H., Mann, I., \& Jessberger, E. K. 2003, ApJ, 582, 846. Elemental abundances and mass densities of dust and gas in the Local IS Cloud

Lu, N., Helou, G., Werner, M. W., et al. 2003, ApJ, 588, 199. Infrared emission of normal galaxies from 2.5 to $12 \mu \mathrm{m}$ : ISO spectra, near-IR continuum, and mid-IR emission features

Savaglio, S., Fall, S. M., \& Fiore, F. 2003, ApJ, 585, 638. Heavy-element abundances and dust depletions in the host galaxies of three $\gamma$-ray bursts

Weingartner, J. C. \& Murray, N. 2002, ApJ, 580, 88. X-ray vs. optical observations of active galactic nuclei: evidence for large grains?

Dupac, X., Giard, M., Bernard, J.-P., et al. 2002, A\&A, 392, 691. Sub-millimeter dust emission of the M 17 complex measured with PRONAOS

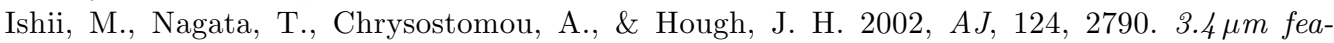
ture on the shoulder of ice-band absorptions in three luminous young stellar objects: IRAS $18511+0146$, IRAS 21413+5442, and IRAS 04579+4703

Boogert, A. C. A., Blake, G. A., Tielens, A. G. G. M. 2002, ApJ, 577, 271. High-resolution $4.7 \mu \mathrm{m}$ Keck/NIRSPEC spectra of protostars. II. Detection of the ${ }^{13} \mathrm{CO}$ isotope in icy grain mantles

Chiar, J. E., Adamson, A. J., Pendleton, Y. J., et al. 2002, ApJ, 570, 209. Hydrocarbons, ices, and ' $X C N$ ' in the line of sight toward the Galactic Center

Pendleton, Y. J. \& Allamandola, L. J. 2002, ApJS, 138, 75. The organic refractory material in the diffuse IS medium: mid-IR spectroscopic constraints

\section{Properties of dust}

\section{References}

Thompson, S. P. 2008, A\&A A, 484, 251. Structural signatures of medium-range order in annealed laboratory silicates

Mason, N. J., Drage, E. A., Webb, S. M., et al. 2008, Faraday Discuss., 137, 367. The spectroscopy and chemical dynamics of micro-particles explored using an ultrasonic trap

Whittet, D. C. B., Hough, J. H., Lazarian, A., \& Hoang, T. 2008, ApJ, 674, 304. The efficiency of grain alignment in dense IS clouds: a re-assessment of constraints from near-IR polarization

Iatì, M. A., Saija, R., Borghese, F., et al. 2008, MNRAS, 384, 591. Stratified dust grains in the IS medium. I. An accurate computational method for calculating their optical properties

Lazzati, D. 2008, MNRAS, 384, 165. Non-LTE dust nucleation in sub-saturated vapours

Rosenberg, J. L., Wu, Y., Le Floc'h, E., et al. 2008, ApJ, 674, 814. Dust properties and starformation rates in star-forming dwarf galaxies 
Engelbracht, C. W., Rieke, G. H., Gordon, K. D., et al. 2008, ApJ, 678, 804. Metallicity effects on dust properties in starbursting galaxies

Voshchinnikov, N. V., \& Henning, T. 2008, A $E A$ (Letters), 483, L9. Is the silicate emission feature only influenced by grain size?

Jiménez-Serra, I., Caselli, P., Martín-Pintado, J., Hartquist, T. W. 2008, A\& A, 482, 549. Parametrization of $C$-shocks. Evolution of the sputtering of grains

Pitman, K. M., Hofmeister, A. M., Corman, A. B., \& Speck, A. K. 2008, A\& A, 483, 661. Optical properties of silicon carbide for astrophysical applications. I. New laboratory IR reflectance spectra and optical constants

Calura, F., Pipino, A., \& Matteucci, F. 2008, A\&A A, 479, 669. The cycle of IS dust in galaxies of different morphological types

Weingartner, J. C. \& Jordan, M. E. 2008, ApJ, 672, 382. Torques on spheroidal silicate grains exposed to anisotropic IS radiation fields

Fabian, A. C., Vasudevan, R. V., \& Gandhi, P. 2008, MNRAS (Letters), 385, L43. The effect of radiation pressure on dusty absorbing gas around active galactic nuclei

Lazarian, A. \& Hoang, T. 2008, ApJ (Letters), 676, L25. Alignment of dust with magnetic inclusions: radiative torques and superparamagnetic Barnett and nuclear relaxation

Nozawa, T., Kozasa, T., Habe, A., et al. 2008, in: First Stars III AIP-CP, 990, 426. Dust evolution in Pop. III supernova remnants

Zhukovska, S., Gail, H.-P., \& Trieloff, M. 2008, AESA, 479, 453. Evolution of IS dust and stardust in the solar neighbourhood

Guillet, V., Pineau Des Forêts, G., \& Jones, A. P. 2007, A\&A, 476, 263. Shocks in dense clouds. I. Dust dynamics

Dartois, E. 2007, in: D. C. Lis, G. A. Blake \& E. Herbst (eds.), Astrochemistry throughout the Universe: Recent Successes and Current Challenges, Proc. IAU Symposium No. 231 (Cambridge: CUP), p. 54. IS dust grains: the hydrogenated amorphous carbon contribution

Wada, K., Tanaka, H., Suyama, T., et al. 2007, ApJ, 661, 320. Numerical simulation of dust aggregate collisions. I. Compression and disruption of 2-D aggregates

Draine, B. T., Li, A. 2007, ApJ, 657, 810. Infrared emission from IS dust. IV. The silicategraphite-PAH model in the post-Spitzer era

Ferguson, J. W., Heffner-Wong, A., Penley, J. J., et al. 2007, ApJ, 666, 261. Grain physics and Rosseland-mean opacities

Vaidya, D. B., Gupta, R., \& Snow, T. P. 2007, MNRAS, 379, 800. Composite IS grains

Miville-Deschênes, M.-A., Lagache, G., Boulanger, F., \& Puget, J.-L. 2007, A\&\&A, 469, 595. Statistical properties of dust far-IR emission

Maheswar, G., Muthu, C., Sujatha, N. V., et al. 2007, BASI, 35, 233. IS dust studies with TAUVEX

Djouadi, Z., Gattacceca, J., D’Hendecourt, L., et al. 2007, A\&A (Letters), 468, L9. Ferromagnetic inclusions in silicate thin films: insights into the magnetic properties of cosmic grains

Huss, G. R. \& Draine, B. T. 2007, in: K. A. van der Hucht (ed.), Highlights of Astronomy, 14, 353. What can pre-solar grains tell us about the Solar nebula?

Nguyen, A. N., Stadermann, F. J., Zinner, E., et al. 2007, ApJ, 656, 1223. Characterization of pre-solar silicate and oxide grains in primitive carbonaceous chondrites

Ott, U., Hoppe, P. 2007. Pre-solar Grains in Meteorites and Interplanetary Dust: an Overview. Highlights of Astronomy 14, 341-344

Min, M., Waters, L. B. F. M., de Koter, A., et al. 2007, A\&A A, 462, 667. The shape and composition of IS silicate grains

Pelkonen, V.-M., Juvela, M., \& Padoan, P. 2007, A\&AA, 461, 551. Simulations of polarized dust emission

Ormel, C. W., Spaans, M., Tielens, A. G. G. M. 2007, A\&BA, 461, 215. Dust coagulation in protoplanetary disks: porosity matters

Patil, M. K., Pandey, S. K., Sahu, D. K., \& Kembhavi, A. 2007, A\&A, 461, 103. Properties of dust in early-type galaxies

Vaillancourt, J. E. 2007, EAS-PS, 23, 147. Polarized emission from IS dust

Lazarian, A. 2007, JQSRT, 106, 225. Tracing magnetic fields with aligned grains

Ehrenfreund, P., Ruiterkamp, R., Peeters, Z., et al. 2007, PESSS, 55, 383. The ORGANICS Experiment on BIOPAN V: UV and space exposure of aromatic compounds 
Li, M. P., Zhao, G., \& Li, A. 2007, MNRAS (Letters), 382, L26. On the crystallinity of silicate dust in the IS medium

Bianchi, S. \& Schneider, R. 2007, MNRAS, 378, 973. Dust formation and survival in supernova ejecta

Lazarian, A. \& Hoang, T. 2007, MNRAS, 378, 910. Radiative torques: analytical model and basic properties

Abbas, M. M., Tankosic, D., Craven, P. D., et al. 2006, ApJ, 645, 324. Photoelectric emission measurements on the analogs of individual cosmic dust grains

Duley, W. W. 2006, Faraday Discuss., 133, 415. Polycyclic aromatic hydrocarbons, carbon nanoparticles and the diffuse IS bands

Ehrenfreund, P., Ruiterkamp, R., Peeters, Z., et al. 2006, COSPAR, 36, 3635. The ORGANICS Experiments on BIOPAN V:UV and space exposure of aromatic compounds

Freund, M. M. \& Freund, F. T. 2006, ApJ, 639, 210. Solid solution model for IS dust grains and their organics

Altobelli, N., Grün, E., \& Landgraf, M. 2006, A\&A, 448, 243. A new look into the Helios dust experiment data: presence of IS dust inside the Earth's orbit

Stroud, R. M. 2005, in: A. N. Krot, E. R. D. Scott \& B. Reipurth (eds.), Chondrites and the Protoplanetary Disk, ASP-CS, 341, 645. Micro-structural investigations of the cosmochemical histories of pre-solar grains

Kemper, F., Vriend, W. J., \& Tielens, A. G. G. M. 2005, ApJ 633, 534. Erratum: 'The absence of crystalline silicates in the diffuse IS medium', ApJ, 609, 826, 2004

Ruiterkamp, R., Cox, N. L. J., Spaans, M., et al. 2005, A\&A, 432, 515. PAH charge state distribution and DIB carriers: implications from the line of sight toward HD 147889

Tielens, A. G. G. M., Waters, L. B. F. M., \& Bernatowicz, T. J. 2005, in: A. N. Krot, E. R. D. Scott \& B. Reipurth (eds.), Chondrites and the Protoplanetary Disk, ASP-CS, 341, 605. Origin and evolution of dust in circumstellar and IS environments

Dwek, E. 2005, in: Planetary Nebulae as Astronomical Tools, AIP-CP, 804, 197. The chemical evolution of IS dust

Grün, E., Srama, R., Krüger, H., et al. 2005, Icarus, 174, 1. 2002 Kuiper Prize Lecture: Dust Astronomy

Draine, B. T. 2005, ESA-SP, 577, 251. Infrared emission and models of IS dust

Voshchinnikov, N. V., Il'in, V. B., \& Henning, T. 2005, A\&AA, 429, 371. Modelling the optical properties of composite and porous IS grains

Bréchignac, P. \& Schmidt, M. 2005, in: D. C. Lis, G. A. Blake \& E. Herbst (eds.), Astrochemistry throughout the Universe: Recent Successes and Current Challenges, Proc. IAU Symposium No. 231 (Cambridge: CUP), p. 255. From cold gas phase coronene clusters to hydrocarbonated nanograins

Rapacioli, M., Joblin, C., Calvo, F., et al. 2005, in: D. C. Lis, G. A. Blake \& E. Herbst (eds.), Astrochemistry throughout the Universe: Recent Successes and Current Challenges, Proc. IAU Symposium No. 231 (Cambridge: CUP), p.200. Theoretical properties of polycyclic aromatic hydrocarbon clusters of astrophysical interest

Mennella, V. 2005, JPhCS, 6, 197. Dust evolution from the laboratory to the IS medium

van Breugel, W., Bajt, S., Bradley, J., et al. 2005, in: A. Wilson (ed.), The Dusty and Molecular Universe: a Prelude to Herschel and ALMA, ESA-SP, 577, 91. Star formation in highpressure, high-energy density environments: laboratory experiments of ISM dust analogs

Wooden, D. H., Harker, D. E., \& Brearley, A. J. 2005, in: A. N. Krot, E. R. D. Scott \& B. Reipurth (eds.), Chondrites and the Protoplanetary Disk, ASP-CS, 341, 774. Thermal processing and radial mixing of dust: evidence from comets and primitive chondrites

Zinov'Eva, T. V. 2005, AstL, 31, 458. Modeling infrared absorption bands with non-spherical particles

Biennier, L., Hammond, M., Elsila, J., et al. 2005, in: D. C. Lis, G. A. Blake \& E. Herbst (eds.), Astrochemistry throughout the Universe: Recent Successes and Current Challenges, Proc. IAU Symposium No. 231 (Cambridge: CUP), p. 214. From organic molecules to carbon particles: implications for the formation of IS Dust

Keane, J. V., Pendleton, Y. J., \& Allamandola, L. J. 2005, in: D. C. Lis, G. A. Blake \& E. Herbst (eds.), Astrochemistry throughout the Universe: Recent Successes and Current Challenges, 
Proc. IAU Symposium No. 231 (Cambridge: CUP), p. 242. Refractory carbonaceous material in luminous galaxies: mid-IR spectroscopic constraints

Salama, F. \& Biennier, L. 2004, COSPAR, 35, 3067. Formation and destruction processes of IS dust: from organic molecules to carbonaceous grains

Zubko, V., Dwek, E., \& Arendt, R. G. 2004, ApJS, 152, 211. IS dust models consistent with extinction, emission, and abundance constraints

Li, A. 2004. in: A. N. Witt, G. C. Clayton \& B. T. Draine (eds.), Astrophysics of Dust, ASP-CS, 309, 417. Interaction of nanoparticles with radiation

Iatì, M. A., Giusto, A., Saija, R., et al. 2004, ApJ, 615, 286. Optical properties of composite IS grains: a morphological analysis

Jones, A. P. \& D'Hendecourt, L. B. 2004, in: A. N. Witt, G. C. Clayton \& B. T. Draine (eds.), Astrophysics of Dust, ASP-CS, 309, 589. IS nano-diamonds

Shenoy, S. S., Whittet, D. C. B., Chiar, J. E., et al. 2003, ApJ, 591, 962. A test case for the organic refractory model of IS dust

Hegmann, M. \& Kegel, W. H. 2003, MNRAS, 342, 453. Radiative transfer in clumpy environments: absorption and scattering by dust

Gibson, S. J. \& Nordsieck, K. H. 2003, ApJ, 589, 362. The Pleiades reflection nebula. II. Simple model constraints on dust properties and scattering geometry

Stepnik, B., Abergel, A., Bernard, J.-P., et al. 2003, A\&GA, 398, 551. Evolution of dust properties in an IS filament

Juvela, M. \& Padoan, P. 2003, A\&SA, 397, 201. Dust emission from inhomogeneous IS clouds: radiative transfer in 3-D with transiently heated particles

Mann, I. \& Jessberger, E. K. 2003, Astromineralogy, 609, 189. The in-situ study of solid particles in the Solar system

Hanner, M. S. 2003, Astromineralogy 609, 171. The mineralogy of cometary dust

Lazarian, A. 2003, JQSRT 79, 881. Magnetic fields via polarimetry: progress of grain alignment theory

Mathis, J. S., Whitney, B. A., \& Wood, K. 2002, ApJ, 574, 812. Can reflection from grains diagnose the albedo?

Stepnik, B., Abergel, A., Bernard, J. P., et al. 2002, in: M. Giard et al. (eds.), Infrared and Submillimeter Space Astronomy, EAS-PS, 4, 309. Evolution of IS dust properties from diffuse medium to a dense cloud

Stepnik, B., Jones, A. P., Abergel, A., et al. 2002. in: M. Giard et al. (eds.), Infrared and Sub-millimeter Space Astronomy, EAS-PS, 4, 31. Grain-grain co-agulation in the ISM

Ruiterkamp, R., Ehrenfreund, P., et al. 2002, in: F. Salama (ed.), NASA Laboratory Astrophysics Workshop, NASA/CP-2002-21186, p.149. Laboratory calibration studies in support of ORGANICS on the International Space Station: evolution of organic matter in space

Cox, N., Ehrenfreund, P., Cami, J., et al. 2002, in: H. Lacoste (ed.), Proc. First European Workshop on Exo-Astrobiology, ESA-SP 518, 447. Complex carbon chemistry and the diffuse IS bands in the Magellanic Clouds

\section{Interactions of atoms and molecules with solids in simulated ISM conditions}

\section{References}

Luna, R., Millán, C., Domingo, M., \& Satorre, M. Á. 2008, ApछSS, 314, 113. Thermal desorption of $\mathrm{CH}_{4}$ retained in $\mathrm{CO}_{2}$ ice

Cazaux, S., Caselli, P., Cobut, V., \& Le Bourlot, J. 2008, A\&3A, 483, 495. The role of carbon grains in the deuteration of $\mathrm{H}_{2}$

Vidali, G., Pirronello, V., Li, L., et al. 2007, JPhChA, 111, 12611. Analysis of molecular hydrogen formation on low temperature surfaces in temperature programmed desorption experiments

Li, L., Manico, G., Congiu, E., et al. 2007, in: J. L. Lemaire \& F. Combes (eds.), Molecules in Space and Laboratory (Paris: S. Diana), p. 58. Formation of molecular hydrogen on amorphous silicate surfaces

Al-Halabi, A., van Dishoeck, E. F., 2007, MNRAS, 382, 1648. Hydrogen adsorption and diffusion on amorphous solid water ice 
Roberts, J. F., Rawlings, J. M. C., Viti, S., \& Williams, D. A. 2007, MNRAS, 382, 733. Desorption from IS ices

Perets, H. B., Lederhendler, A., Biham, O., et al. 2007, ApJ (Letters), 661, L163. Molecular hydrogen formation on amorphous silicates under IS conditions

Bouwman, J., Ludwig, W., Awad, Z., et al. 2007, A\&A, 476, 995. Band profiles and band strengths in mixed $\mathrm{H}_{2} \mathrm{O}: \mathrm{CO}$ ices

Öberg, K. I., Fuchs, G. W., Awad, Z., et al. 2007, ApJ (Letters), 662, L23. Photodesorption of CO ice

Chaabouni, H., Amiaud, L., Dulieu, F., et al. 2007, in: J. L. Lemaire \& F. Combes (eds.), Molecules in Space and Laboratory (Paris: S. Diana), p. 67. Sticking of deuterium molecules onto non-porous water ice surface: temperature dependence of the impinging molecules

Chang, Q., Cuppen, H. M., \& Herbst, E. 2007, A\&A, 469, 973. Gas-grain chemistry in cold IS cloud cores with a microscopic Monte Carlo approach to surface chemistry

Watanabe, N., Mouri, O., et al. 2007, ApJ, 668, 1001. Laboratory simulation of competition between hydrogenation and photolysis in the chemical evolution of $\mathrm{H}_{2} \mathrm{O}-\mathrm{CO}$ ice mixtures

Canto, J., Gomis, O., Vilaplana, R., \& Domingo, M. 2007, in: J. L. Lemaire \& F. Combes (eds.), Molecules in Space and Laboratory (Paris: S. Diana), p. 65. A laboratory for studying simple ices and their mixtures in the far-IR region

Bisschop, S. E., Fuchs, G. W., van Dishoeck, E. F., \& Linnartz, H. 2007, A\&A, 474, 1061. $\mathrm{H}$-atom bombardment of $\mathrm{CO}_{2}, \mathrm{HCOOH}$, and $\mathrm{CH}_{3} \mathrm{CHO}$ containing ices

Xie, H.-B., Shao, C.-B., \& Ding, Y.-H., 2007, ApJ, 670, 449. Radical-molecule reaction $C_{3} H+$ $\mathrm{H}_{2} \mathrm{O}$ on amorphous water ice: a promising route for IS propynal

Amiaud, L., Dulieu, F., Fillion, J.-H., et al. 2007, JChPh, 127, 4709. Interaction of atomic and molecular deuterium with a non-porous amorphous water ice surface between 8 and $30 \mathrm{~K}$

Goldsmith, P.F. 2007, in: J. L. Lemaire \& F. Combes (eds.), Molecules in Space and Laboratory (Paris: S. Diana), p.93. Conversion of $\mathrm{HI}$ to $\mathrm{H}_{2}$ and the age of molecular clouds

Goumans, T. P. M., Wander, A., Catlow, C. R. A., \& Brown, W. A. 2007, MNRAS, 382, 1829. Silica grain catalysis of methanol formation

Elsila, J. E., Dworkin, J. P., Bernstein, M. P., et al. 2007, Mechanisms of amino acid formation in IS ice analogs ApJ, 660, 911

Bar-Nun, A., Notesco, G., \& Owen, T. 2007, Icarus, 190, 655. Trapping of $N_{2}, C O$ and Ar in amorphous ice application to comets

Öberg, K. I., Fraser, H. J., Boogert, A. C. A., et al. 2007, A\&SA, 462, 1187. Effects of $\mathrm{CO}_{2}$ on $\mathrm{H}_{2} \mathrm{O}$ band profiles and band strengths in mixed $\mathrm{H}_{2} \mathrm{O}: \mathrm{CO}_{2}$ ices

Brown, W. A. \& Bolina, A. S. 2007, MNRAS, 374, 1006. Fundamental data on the desorption of pure IS ices

Hidaka, H., Kouchi, A., \& Watanabe, N. 2007, JChPh, 126, 204707. Temperature, composition, and hydrogen isotope effect in the hydrogenation of $C O$ on amorphous ice surface at 10-20 K

Gálvez, O., Ortega, I. K., Maté, B., et al. 2007, AछA, 472, 691. A study of the interaction of $\mathrm{CO}_{2}$ with water ice

Acharyya, K., Fuchs, G. W., Fraser, H. J., et al. 2007, A\&A, 466, 1005. Desorption of CO and $\mathrm{O}_{2}$ IS ice analogs

Garrod, R. T., Wakelam, V., \& Herbst, E. 2007, A\&A, 467, 1103. Non-thermal desorption from IS dust grains via exo-thermic surface reactions

Xie, H.-B., Shi, G.-S., \& Ding, Y.-H. 2007, ApJ, 662, 758. Chemical behavior of polycyanoacetylene radicals on gaseous and ice water: a computational perspective

Linnartz, H., Acharyya, K., Awad, Z., et al. 2007, in: J. L. Lemaire \& F. Combes (eds.), Molecules in Space and Laboratory (Paris: S. Diana), p. 47. Solid state astrophysics and-Chemistry four Questions- four answers

Bisschop, S. E., Fuchs, G. W., Boogert, A. C. A., et al. 2007, A $₫$ A, 470, 749. Infrared spectroscopy of $\mathrm{HCOOH}$ in IS ice analogues

Bernstein, M. P., Sandford, S. A., Mattioda, A. L., \& Allamandola, L. J. 2007, ApJ, 664, 1264. Near- and mid-IR laboratory spectra of PAH cations in solid $\mathrm{H}_{2} \mathrm{O}$

Barzel, B. \& Biham, O. 2007, ApJ (Letters), 658, L37. Efficient simulations of IS gas-grain chemistry using moment equations

Cuppen, H. M. \& Herbst, E. 2007, ApJ, 668, 294. Simulation of the formation and morphology of ice mantles on IS grains 
Xie, H.-B., Ding, Y.-H., \& Sun, C.-C. 2006, ApJ, 643, 573. Reaction mechanism of oxygen atoms with cyanoacetylene in the gas phase and on water ice

Brown, W. A., Viti, S., Wolff, A. J., \& Bolina, A. S. 2006, Faraday Discuss., 133, 113. Laboratory investigations of the role of the grain surface in astrochemical models

Ehrenfreund, P. \& Sephton, M. A. 2006, Carbon molecules in space: from astrochemistry to astrobiology Faraday Discuss. 133, 277

Garrod, R., Park, I. H., Caselli, P., \& Herbst, E. 2006, Faraday Discuss., 133, 51. Are gas-phase models of IS chemistry tenable? The case of methanol

Madzunkov, S., Shortt, B. J., MacAskill, J. A., et al. 2006, PRA, 73, 020901. Measurements of polyatomic molecule formation on an icy grain analog using fast atoms

Hiraoka, K., Mochizuki, N., \& Wada, A. 2006, in: Astrochemistry - from Laboratory Studies to Astronomical Observations, AIP-CP, 855, 86. How are $\mathrm{CH}_{3} \mathrm{OH}, \mathrm{HNC} / \mathrm{HCN}$, and $\mathrm{NH}_{3}$ formed in the IS Medium?

van Dishoeck, E. F., Acharyya, K., Al-Halabi, A., et al. 2006, in: Astrochemistry - from Laboratory Studies to Astronomical Observations AIP-CP, 855, 113. Spectroscopy and processing of IS ice analogs

Peeters, Z., Rodgers, S. D., Charnley, S. B., et al. 2006, AछA, 445, 197. Astrochemistry of dimethyl ether

Bisschop, S. E., Fraser, H. J., Öberg, K. I., et al. 2006, A\&A, 449, 1297. Desorption rates and sticking coefficients for $\mathrm{CO}$ and $\mathrm{N}_{2}$ IS ices

Collings, M. P., Chen, R., \& McCoustra, M. R. S. 2006, in: Astrochemistry - from Laboratory Studies to Astronomical Observations AIP-CP, 855, 62. Probing the morphology of IS ice analogues

Amiaud, L., Fillion, J. H., Baouche, S., et al. 2006, JChPh, 124, 4702. Interaction of $D_{2}$ with $\mathrm{H}_{2} \mathrm{O}$ amorphous ice studied by temperature-programed desorption experiments

Vidali, G., Roser, J. E., Li, L., et al. 2006, Faraday Discuss., 133, 125. The formation of IS molecules via reactions on dust grain surfaces

Watanabe, N., Hidaka, H., \& Kouchi, A. 2006, in: Astrochemistry - from Laboratory Studies to Astronomical Observations AIP-CP, 855, 122. Relative reaction rates of hydrogenation and deuteration of solid CO at very low temperatures

Woon, D. E. 2006, in: Astrochemistry - from Laboratory Studies to Astronomical Observations AIP-CP $, 855,305$. Ab initio quantum chemical studies of reactions in astrophysical ices reactions involving $\mathrm{CH}_{3} \mathrm{OH}, \mathrm{CO}_{2}, \mathrm{CO}$, and $\mathrm{HNCO}$ in $\mathrm{H}_{2} \mathrm{CO} / \mathrm{NH}_{3} / \mathrm{H}_{2} \mathrm{O}$ ices

Fraser, H. J., Bisschop, S. E., Pontoppidan, K. M., et al. 2005, MNRAS, 356, 1283. Probing the surfaces of IS dust grains: the adsorption of $C O$ at bare grain surfaces

Lipshtat, A. \& Biham, O. 2005, MNRAS, 362, 666. The effect of grain size distribution on $\mathrm{H}_{2}$ formation rate in the IS medium

Collings, M. P., Dever, J. W., McCoustra, \& M. R. S. 2005, CPL, 415, 40. Sub-monolayer coverages of $\mathrm{CO}$ on water ice

Öberg, K. I., van Broekhuizen, F., Fraser, H. J., et al. 2005, ApJ (Letters), 621, L33. Competition between $\mathrm{CO}$ and $\mathrm{N}_{2}$ desorption from IS ices

Bisschop, S. E., Fraser, H. J., Fuchs, G., et al. 2005, in: D. C. Lis, G. A. Blake \& E. Herbst (eds.), Astrochemistry throughout the Universe: Recent Successes and Current Challenges, Proc. IAU Symposium No. 231 (Cambridge: CUP), p. 168. The behavior of $\mathrm{N}_{2}$ and $\mathrm{O}_{2}$ in pure, mixed or layered $C O$ ices

Roberts, H. 2005, in: D. C. Lis, G. A. Blake \& E. Herbst (eds.), Astrochemistry throughout the Universe: Recent Successes and Current Challenges, Proc. IAU Symposium No. 231 (Cambridge: CUP), p. 27. Modelling of deuterium chemistry in star-forming regions

Charnley, S. B. \& Rodgers, S. D. 2005, in: D. C. Lis, G. A. Blake \& E. Herbst (eds.), Astrochemistry throughout the Universe: Recent Successes and Current Challenges, Proc. IAU Symposium No. 231 (Cambridge: CUP), p. 237. Pathways to molecular complexity

Hornekaer, L., Baurichter, A., Petrunin, V. V., \& Luntz, A. C. 2005, ESA-SP, 577, 369. The influence of dust grain morphology on $\mathrm{H}_{2}$ formation and desorption in the IS medium

Fraser, H. J., Bisschop, S. E., Pontoppidan, K. M., et al. 2005, MNRAS, 356, 1283. Probing the surfaces of IS dust grains: the adsorption of $C O$ at bare grain surfaces

Woon, D. E. \& Park, J.-Y. 2005, in: D. C. Lis, G. A. Blake \& E. Herbst (eds.), Astrochemistry throughout the Universe: Recent Successes and Current Challenges, Proc. IAU Symposium 
No. 231 (Cambridge: CUP), p. 89. A density functional theory study of the formation and spectroscopy of the formate $\left(\mathrm{HCOO}^{-}\right)$and ammonium $\left(\mathrm{NH}_{4}^{+}\right)$ions in IS ices

Cuppen, H. M. \& Herbst, E. 2005, in: D. C. Lis, G. A. Blake \& E. Herbst (eds.), Astrochemistry throughout the Universe: Recent Successes and Current Challenges, Proc. IAU Symposium No. 231 (Cambridge: CUP), p. 34. Molecular hydrogen formation on IS grains

Fillion, J.-H., Amiaud, L., Dulieu, F., et al. 2005, in: D. C. Lis, G. A. Blake \& E. Herbst (eds.), Astrochemistry throughout the Universe: Recent Successes and Current Challenges, Proc. IAU Symposium No. 231 (Cambridge: CUP), p. 101. Experimental studies of $\mathrm{H}_{2}$ and $\mathrm{D}_{2}$ interaction with water ice films

Wolff, A. J. \& Brown, W. A. 2005, in: D. C. Lis, G. A. Blake \& E. Herbst (eds.), Astrochemistry throughout the Universe: Recent Successes and Current Challenges, Proc. IAU Symposium No. 231 (Cambridge: CUP), p.57. Mixed methanol/water ice on cosmic dust grain analogues

Vidali, G., Roser, J. E., Manicó, G., \& Pirronello, V. 2005, in: D. C. Lis, G. A. Blake \& E. Herbst (eds.), Astrochemistry throughout the Universe: Recent Successes and Current Challenges, Proc. IAU Symposium No. 231 (Cambridge: CUP), p. 355. Molecular hydrogen formation on dust grains: a summary of experimental results on molecular hydrogen formation on dust grain analogues

Perets, H. B., Biham, O., Manicó, G., et al. 2005, ApJ, 627, 850. Molecular hydrogen formation on ice under IS conditions

Vidali, G., Roser, J. E., Manicó, et al. 2005, JPhCS, 6, 36. Formation of molecular hydrogen on analogues of IS dust grains: experiments and modelling

Fraser, H. J., Bisschop, S. E., Pontoppidan, K. M., et al. 2005, in: D. C. Lis, G. A. Blake \& E. Herbst (eds.), Astrochemistry throughout the Universe: Recent Successes and Current Challenges, Proc. IAU Symposium No. 231 (Cambridge: CUP), p. 31. CO chemisorbed on bare grain surfaces: the potential for heterogeneous chemistry

Amiaud, L., Baouche, S., Dulieu, F., et al. 2004, in: F. Combes et al. (eds.), SF2A-2004: Semaine de l'Astrophysique Francaise (EdP-CS), p. 487. $D_{2}$ sticking coefficient and desorption rate on various forms of water ice films under IS conditions

Al-Halabi, A., Fraser, H., Kroes, G. J., \& van Dishoeck, E. F. 2004, A\&广A, 422, 777. Adsorption of $C O$ on amorphous water-ice surfaces

Sandford, S. A., Bernstein, M. P., \& Allamandola, L. J. 2004, ApJ, 607, 346. The mid-IR laboratory spectra of naphthalene $\left(\mathrm{C}_{10} \mathrm{H}_{8}\right)$ in Solid $\mathrm{H}_{2} \mathrm{O}$

Vidali, G., Roser, J. E., Manicó, G., \& Pirronello, V. 2004, JGRE, 109, E07S14. Laboratory studies of formation of molecules on dust grain analogues under ISM conditions

Lipshtat, A., Biham, O., \& Herbst, E. 2004, MNRAS, 348, 1055. Enhanced production of HD and $\mathrm{D}_{2}$ molecules on small dust grains in diffuse clouds

Morisset, S., Aguillon, F., Sizun, M., \& Sidis, V. 2003, PCCP, 5, 506. The dynamics of $\mathrm{H}_{2}$ formation on a graphite surface at low temperature

Lipshtat, A. \& Biham, O., 2003, A\&A, 400, 585. Moment equations for chemical reactions on IS dust grains

Meierhenrich, U. J., Muñoz Caro, G. M., Schutte, W. A., et al. 2002, Exo-Astrobiology, 518, 25. The prebiotic synthesis of amino acids - IS vs. atmospheric mechanisms

Woon, D. E. 2002, ApJ, 569, 541. Modeling gas-grain chemistry with quantum chemical cluster calculations. I. Heterogeneous hydrogenation of $\mathrm{CO}$ and $\mathrm{H}_{2} \mathrm{CO}$ on icy grain mantles

Basiuk, V. A. \& Bogillo, V. I. 2002, AdSpR, 30, 1439. Theoretical study of amino acid precursor formation in the IS medium. I. Reaction of methylenimine with hydrogen cyanide

\section{Interaction of radiation and charged particles with ices in simulated ISM conditions}

\section{References}

Chen, Y.-J., Nuevo, M., Yih, T.-S., et al. 2008, MNRAS, 384, 605. Amino acids produced from the UV/EUV irradiation of naphthalene in a $\mathrm{H}_{2} \mathrm{O}+\mathrm{NH}_{3}$ ice mixture

Nuevo, M., Auger, G., Blanot, D., \& D'Hendecourt, L. 2008, OLEB, 38, 37. A detailed study of the amino acids produced from the vacuum UV irradiation of IS ice analogs 
Zheng, W., Jewitt, D., Osamura, Y., \& Kaiser, R. I. 2008, ApJ, 674, 1242. Formation of nitrogen and hydrogen-bearing molecules in solid ammonia and implications for Solar system and IS ices

Thrower, J. D., Burke, D. J., Collings, M. P., et al. 2008, ApJ, 673, 1233. Desorption of hot molecules from photon irradiated IS ices

Davoisne, C., Leroux, H., Frère, M., et al. 2008, A\&A, 482, 541. Chemical and morphological evolution of a silicate surface under low-energy ion irradiation

Chen, Y.-J., Nuevo, M., Hsieh, J.-M., et al. 2007, A\&SA, 464, 253. Carbamic acid produced by the UV/EUV irradiation of IS ice analogs

Bringa, E. M., Kucheyev, S. O., Loeffler, M. J., et al. 2007, ApJ, 662, 372. Energetic processing of IS silicate grains by cosmic rays

Zheng, W. \& Kaiser, R. I. 2007, CP, 450, 55. On the formation of carbonic acid $\left(\mathrm{H}_{2} \mathrm{CO}_{3}\right)$ in Solar system ices

Bennett, C. J. \& Kaiser, R. I. 2007, ApJ, 660, 1289. The formation of acetic acid $\left(\mathrm{CH}_{3} \mathrm{COOH}\right)$ in IS ice analogs

Bennett, C. J. \& Kaiser, R. I. 2007, ApJ, 661, 899. On the formation of glycolaldehyde $\left(\mathrm{HCOCH}_{2} \mathrm{OH}\right)$ and methyl formate $\left(\mathrm{HCOOCH}_{3}\right)$ in IS ice analogs

Sivaraman, B., Jamieson, C. S., Mason, N. J., \& Kaiser, R. I. 2007, ApJ, 669, 1414. Temperaturedependent formation of ozone in solid oxygen by $5 \mathrm{keV}$ electron irradiation and implications for Solar system ices

Wada, A., Mochizuki, N., \& Hiraoka, K. 2006, ApJ, 644, 300. Methanol formation from electronirradiated mixed $\mathrm{H}_{2} \mathrm{O} / \mathrm{CH}_{4}$ ice at $10 \mathrm{~K}$

Ricca, A., Bakes, E. L. O., Bauschlicher, C. W. 2007, ApJ, 659, 858. The energetics for hydrogen addition to naphthalene cations

Öberg, K. I., Fuchs, G. W., Awad, Z., et al. 2007, ApJ (Letters), 662, L23. Photodesorption of CO ice

Schriver, A., Schriver-Mazzuoli, L., Ehrenfreund, P., \& D'Hendecourt, L. 2007, CP, 334, 128. One possible origin of ethanol in IS medium: photochemistry of mixed $\mathrm{CO}_{2} \mathrm{C}_{2} \mathrm{H}_{6}$ films at 11 K. A FTIR study

Mason, N. J., Dawes, A., Holtom, P. D., et al. 2006, in: Astrochemistry - From Laboratory Studies to Astronomical Observations AIP-CP, 855, 128. VUV spectroscopy of extraterrestrial ices

Loeffler, M. J., Raut, U., Vidal, R. A., et al. 2006, Icarus, 180, 265. Synthesis of hydrogen peroxide in water ice by ion irradiation

Jamieson, C. S., Mebel, A. M., \& Kaiser, R. I. 2006, ApJS, 163, 184. Understanding the kinetics and dynamics of radiation-induced reaction pathways in carbon monoxide ice at $10 \mathrm{~K}$

Palumbo, M. E. 2006, A\&A A, 453, 903. Formation of compact solid water after ion irradiation at $15 K$

Jamieson, C. S., Guo, Y., Gu, X., et al. 2006, in: F. Salama (ed.), NASA Laboratory Astrophysics Workshop, NASA/CP-2002-21186, p.68. Laboratory studies on the formation of carbonbearing molecules in extraterrestrial environments: from the gas phase to the solid state

Wooden, D. H., Harker, D. E., \& Brearley, A. J. 2005, in: A. N. Krot, E. R. D. Scott \& B. Reipurth (eds.) Chondrites and the Protoplanetary Disk ASP-CS, 341, 774. Thermal processing and radial mixing of dust: evidence from comets and primitive chondrites

Bernstein, M. P., Sandford, S. A., \& Allamandola, L. J. 2005, ApJS, 161, 53. The mid-IR absorption spectra of neutral PAHs in conditions relevant to dense IS clouds

Moore, M. H. \& Hudson, R. L. 2005, in: D. C. Lis, G. A. Blake \& E. Herbst (eds.), Astrochemistry throughout the Universe: Recent Successes and Current Challenges, Proc. IAU Symposium No. 231 (Cambridge: CUP), p. 247. Production of complex molecules in astrophysical ices

Farenzena, L. S., Iza, P., Martinez, R., et al. 2005, EMESP, 97, 311. Electronic sputtering analysis of astrophysical ices

Hudson, R. L., Khanna, R. K., \& Moore, M. H. 2005, ApJS, 159, 277. Laboratory evidence for solid-phase protonation of HNCO in IS ices

Holtom, P. D., Bennett, C. J., Osamura, Y., et al. 2005, ApJ, 626, 940. A combined experimental and theoretical study on the formation of the amino acid glycine $\left(\mathrm{NH}_{2} \mathrm{CH}_{2} \mathrm{COOH}\right)$ and its isomer $\left(\mathrm{CH}_{3} \mathrm{NHCOOH}\right)$ in extraterrestrial ices 
Bernstein, M. P., Mattioda, A. L., Sandford, S. A., \& Hudgins, D. M. 2005, ApJ, 626, 909. Laboratory IR spectra of polycyclic aromatic nitrogen heterocycles: quinoline and phenanthridine in solid argon and $\mathrm{H}_{2} \mathrm{O}$

Shalabiea, O. M., Awad, Z., Chigai, T., \& Yamamoto, T. 2005, in: D. C. Lis, G. A. Blake \& E. Herbst (eds.), Astrochemistry throughout the Universe: Recent Successes and Current Challenges, Proc. IAU Symposium No. 231 (Cambridge: CUP), p. 64. New rate constants of hydrogenation on IS grains and their astrophysical implications

Creighan, S. C. \& Price, S. D. 2005, in: D. C. Lis, G. A. Blake \& E. Herbst (eds.), Astrochemistry throughout the Universe: Recent Successes and Current Challenges, Proc. IAU Symposium No. 231 (Cambridge: CUP), p. 55. Studies of hydrogen formation on IS grain analogues

Strazzulla, G. \& Moroz, L. 2005, A\& A, 434, 593. Ion irradiation of asphaltite as an analogue of solid hydrocarbons in the IS medium

Mukerji, R. J., Dawes, A., Holtom, P. D., et al. 2005, in: D. C. Lis, G. A. Blake \& E. Herbst (eds.), Astrochemistry throughout the Universe: Recent Successes and Current Challenges, Proc. IAU Symposium No. 231 (Cambridge: CUP), p. 188. Studies of the temperature dependence of the photo-absorption spectrum of solid ammonia

Strazzulla, G., Leto, G., LaDelfa, S., et al. 2005, MemSAIS, 6, 51. Oxidants produced after ion bombardment of water/carbon dioxide icy mixtures

Holtom, P. D., Dawes, A., Davis, M. P., et al. 2005, in: D. C. Lis, G. A. Blake \& E. Herbst (eds.), Astrochemistry throughout the Universe: Recent Successes and Current Challenges, Proc. IAU Symposium No. 231 (Cambridge: CUP), p. 130. VUV photo-absorption spectroscopy of amorphous and crystalline sulphur dioxide films

Davis, M. P., Dawes, A., Holtom, P. D., et al. 2005, in: D. C. Lis, G. A. Blake \& E. Herbst (eds.), Astrochemistry throughout the Universe: Recent Successes and Current Challenges, Proc. IAU Symposium No. 231 (Cambridge: CUP), p.128. Vacuum UV spectroscopy of laboratory-simulated astrophysical ices

Dawes, A., Holtom, P. D., Mukerji, R. J., et al. 2005, in: D. C. Lis, G. A. Blake \& E. Herbst (eds.), Astrochemistry throughout the Universe: Recent Successes and Current Challenges, Proc. IAU Symposium No. 231 (Cambridge: CUP), p. 131. Low-energy singly and multiply charged ion irradiation of astrophysical ices

Gomis, O. \& Strazzulla, G. 2005, Icarus, 177, 570. $\mathrm{CO}_{2}$ production by ion irradiation of $\mathrm{H}_{2} \mathrm{O}$ ice on top of carbonaceous materials and its relevance to the galilean satellites

Hudson, R. L., Moore, M. H., \& Cook, A.M. 2005, AdSpR, 36, 184. IR characterization and radiation chemistry of glycolaldehyde and ethylene glycol ices

Kroes, G. J. \& Andersson, S. 2005, in: D. C. Lis, G. A. Blake \& E. Herbst (eds.), Astrochemistry throughout the Universe: Recent Successes and Current Challenges, Proc. IAU Symposium No. 231 (Cambridge: CUP), p. 427. Theory of molecular scattering from and photo-chemistry at ice surfaces

Palumbo, M. E. 2005, in: D. C. Lis, G. A. Blake \& E. Herbst (eds.), Astrochemistry throughout the Universe: Recent Successes and Current Challenges, Proc. IAU Symposium No. 231 (Cambridge: CUP), p. 59. Formation of compact solid water after cosmic ion irradiation

Mennella, V., Baratta, G. A., Palumbo, M. E., \& Bergin, E. A. 2006, ApJ, 643, 923. Synthesis of $\mathrm{CO}$ and $\mathrm{CO}_{2}$ molecules by UV irradiation of water ice-covered hydrogenated carbon grains

Loeffler, M. J., Raut, U., Vidal, R. A., et al. 2006, Icarus, 180, 265. Synthesis of hydrogen peroxide in water ice by ion irradiation

Hudson, R. L. \& Moore, M. H. 2004, Icarus, 172, 466. Reactions of nitriles in ices relevant to Titan, comets, and the IS medium: formation of cyanate ion, ketenimines, and isonitriles

Palumbo, M. E., 2005, JPhCS, 6, 211. The morphology of IS water ice

Moore, M. H. \& Hudson, R. L. 2005, in: D. C. Lis, G. A. Blake \& E. Herbst (eds.), Astrochemistry throughout the Universe: Recent Successes and Current Challenges, Proc. IAU Symposium No. 231 (Cambridge: CUP), p. 247. Production of complex molecules in astrophysical ices

Tatischeff, V. \& Kiener, J. 2004, NwARv, 48, 99. Gamma-ray lines from cosmic-ray interactions with IS dust grains

Mennella, V., Palumbo, M. E., \& Baratta, G. A. 2004, ApJ, 615, 1073. Formation of CO and $\mathrm{CO}_{2}$ molecules by ion irradiation of water ice-covered hydrogenated carbon grains

Gerakines, P. A., Moore, M. H., Hudson, R. L. 2004, Icarus, 170, 202. UV photolysis and proton irradiation of astrophysical ice analogs containing hydrogen cyanide 
Gomis, O., Leto, G., \& Strazzulla, G. 2004, A\&3A, 420, 405. Hydrogen peroxide production by ion irradiation of thin water ice films

Sandford, S. A., Bernstein, M. P., \& Allamandola, L. J. 2004, ApJ, 607, 346. The mid-IR laboratory spectra of naphthalene $\left(\mathrm{C}_{10} \mathrm{H}_{8}\right)$ in solid $\mathrm{H}_{2} \mathrm{O}$

Muñoz Caro, G. M., Meierhenrich, U., Schutte, W. A., et al. 2004, A\&SA, 413, 209. UV-photoprocessing of IS ice analogs: detection of hexamethylenetetramine-based species

Baratta, G. A., Brunetto, R., Leto, G., et al. 2004, MemSAIS, 5, 33. Ion irradiation of ices relevant to astrophysics

Woon, D. E. 2004, AdSpR, 33, 44. Photoionization in UV processing of astrophysical ice analogs at cryogenic temperatures

Mennella, V., Baratta, G. A., Esposito, A., et al. 2003, The effects of ion irradiation on the evolution of the carrier of the $3.4 \mu \mathrm{m}$ IS absorption band ApJ, 587, 727

Chilton, D. L., Mohr, R., \& Gerakines, P. 2003, BAAS, 35, 1269. Simulating the effect of UV photolysis on IS ices

Muñoz Caro, G. M., \& Schutte, W. A. 2003, A\&A, 412, 121. UV-photo-processing of IS ice analogs: new IR spectroscopic results

Cooper, J. F., Christian, E. R., Richardson, J. D., \& Wang, C. 2003, EM\&PP, 92, 261. Proton irradiation of Centaur, Kuiper Belt, and Oort Cloud objects at plasma to cosmic ray energy

Wu, C. Y. R., Judge, D. L., Cheng, B.-M., et al. 2003, JGRE, 108, 5032. EUV photolysis of $\mathrm{CO}_{2}-\mathrm{H}_{2} \mathrm{O}$ mixed ices at $10 \mathrm{~K}$.

Mennella, V., Baratta, G. A., Esposito, A., et al. 2003, ApJ, 587, 727. The effects of ion irradiation on the evolution of the carrier of the $3.4 \mu \mathrm{m} I S$ absorption band

Muñoz Caro, G. M., Meierhenrich, U.J., Schutte, W.A., et al. 2002, Nature, 416, 403. Amino acids from UV irradiation of IS ice analogues

Carrez, P., Demyk, K., Cordier, P., et al. 2002, Science, 37, 1599. Low-energy helium ion irradiation-induced amorphization and chemical changes in olivine: insights for silicate dust evolution in the IS medium, meteoritics and planetary

Mennella, V., Brucato, J. R., Colangeli, L., \& Palumbo, P. 2002, AdSpR, 30, 1451. Hydrogenation of carbon grains by exposure to hydrogen atoms: implications for the $3.4 \mu \mathrm{m}$ IS absorption band 